\title{
Parametric study of the Two-Temperature Model for Molecular Dynamics simulations of collisions cascades in $\mathrm{Si}$ and Ge
}

\author{
Thomas Jarrin ${ }^{\mathrm{a}, \mathrm{b}, *}$, Antoine Jay ${ }^{\mathrm{b}}$, Anne Hémeryck ${ }^{\mathrm{b}}$, Nicolas Richard ${ }^{\mathrm{a}}$ \\ ${ }^{a} C E A, D A M, D I F$, F-91297 Arpajon, France \\ ${ }^{b}$ LAAS-CNRS, Université de Toulouse, CNRS, Toulouse, France
}

\begin{abstract}
The sensitivity of collision cascades simulations to the Two-Temperature Model main parameters is investigated by performing an extensive statistical study both in $\mathrm{Si}$ and Ge materials. The purpose is to identify the parameters of the Two-Temperature Model whose impact is the most significant on the cascades properties and to discuss the physical role of each parameter. We demonstrate that the electronic stopping power and electron phonon coupling have a drastic impact both in $\mathrm{Si}$ and Ge but that these two parameters act differently on those two materials due to their distinct thermal properties. We show that the formation of thermal spikes and therefore of amorphous pockets is sensitive to the electronic specific heat. The effects are again very distinct in Si and in Ge. The influence of both the threshold velocity for the stopping power and the electron-phonon coupling activation time are found to be negligible at the considered energies.
\end{abstract}

Keywords: collision cascades, displacement damage, semiconductors, molecular dynamics, two-temperature model, electronic effects

\section{Introduction}

In a highly radiative environment such as space or nuclear plants, micro and optoelectronic components can be subjected to Displacement Damage (DD) effects [1]. DD is described as a perturbation of the crystalline lattice by the interaction with incident energetic particles (neutrons, protons, ions). A similar effect can appear during ion implantation into Si wafers for example, where the incoming ion induces DD in the lattice of the target material. For more than thirty years, Molecular Dynamics (MD) simulations have proven themselves to be a very appropriate tool for the simulations of collision cascades in materials, and thus for the detailed understanding of DD [2, 3]. Collision cascades initiated by a Primary Knock-On Atom (PKA) in $\mathrm{Si}$ and Ge have been extensively studied with $\mathrm{MD}[2,4,5]$, giving a qualitative picture of the mechanisms at stake in those materials.

However, an important aspect of the collision cascades that is often neglected in MD simulations is the presence of electronic excitations and deexcitations. Electronic excitations lead to the kinetic energy loss of moving atoms via the electronic stopping power whereas electronic deexcitations allow a part of the energy stored by the electrons to be fed back to the atoms via the electron-phonon coupling [6]. According to SRIM [7], in Si, a $10 \mathrm{keV}$ self recoil loses $18 \%$ of its energy to electronic excitations, it goes up

\footnotetext{
* Corresponding author

Email address: thomas.jarrin@cea.fr (Thomas Jarrin)
}

to $40 \%$ for a $100 \mathrm{keV}$ self recoil and $66 \%$ for a $500 \mathrm{keV}$ self recoil. Electronic excitations are therefore far from being negligible in the energy range spanned by collision cascades.

The model which has certainly been the most employed for coping with electronic excitations in MD simulations of collision cascades is the Two-Temperature Model (TTM) [8]. The model lies on the splitting of the system into an ionic and an electronic subsystem. Two temperatures are defined based on this splitting: an atomic temperature $T_{a}$ and an electronic temperature $T_{e}$. The electronic temperature follows a standard heat equation whose main parameter is the electronic specific heat $C_{e}$. The electronic and ionic subsystems can exchange energy through the electronic stopping power $\gamma_{s}$, applied only on atoms whose velocities are above a threshold one $v_{0}$, and through the electron-phonon coupling $\gamma_{p}$.

The TTM was initially developed for metals and applied to $\mathrm{Fe}[9], \mathrm{W}$ [10], $\mathrm{Ni}$ and $\mathrm{NiFe} \mathrm{[11]} \mathrm{to} \mathrm{cite} \mathrm{a} \mathrm{few}$ examples. The use of the TTM for band gap materials is still being debated as the charge carriers transport is not explicitly tracked and the band gap not considered [12]. However, Khara et al. have shown in [13] that the band gap effects are accounted for through $C_{e}$. Moreover, the TTM developed by Duffy et al. in [8] has already been applied in its original form with success $[5,13,14]$ or in a slightly revised way $[15,16]$ to band gap materials. In this article, we employ the original version of the TTM developed by Duffy et al. in [8] to Si and Ge in order to provide results and discussions useful for the largest possible range 
of TTM users.

Some recent studies have tested TTM-based models complexified by the addition of new parameters $[11,17]$. We made the choice to stay true to the most employed version of the TTM (i.e. the one developed by Duffy et al. in [8]). The only parameter not present in the Duffy et al. version of the TTM we use is the activation time of the electron-phonon coupling $\left(t_{e p h}\right)$ which acts to delay by a fraction of picosecond the time at which the electronphonon coupling is activated (Zarkadoula et al. set it between $0.2 \mathrm{ps}$ and $1 \mathrm{ps}$ in their works [11, 10, 18]) and whose use is vivedly recommended [11] and well spread in the community [17, 10, 18, 19]. Experiments [20, 21] and MD simulations [2] have showed that the response of $\mathrm{Si}$ and Ge materials to particle irradiation is very unalike because of their different thermal properties [4]. The impact of the TTM in both material is therefore expected to be dissimilar.

Despite the fact that the TTM is becoming common to take into account the electronic effects in MD simulations, very few studies have been entirely dedicated to a systematic study of the effects of the TTM parameters on collision cascades: Khara et al. studied in [13] the effects of $C_{e}$ on Swift Heavy Ions (SHI) irradiation in Si, Zarkadoula et al. explored in [11] the effects of electron-phonon coupling in $\mathrm{Ni}$ and NiFe. In W, Cui et al. studied in [17] the impact of electronic stopping power and Sand et al. explored in [22] and [23] the effects of $v_{0}$ on the production of defects with models similar to the TTM. No extensive statistical study considering large enough number of simulations to overcome the high stochasticity of the cascades has been dedicated to the impact of the TTM parameters on collision cascades, especially in semiconducting materials. Such a study would be very valuable considering the lack of reliable experimental or theoretical values and the uncertainty in the methods to determine some TTM parameters in even simple materials which can compel to make choices by default without always having a clear view on the implications. For instance, in Si and in Ge, apart from $\gamma_{s}$ which can be accurately and easily determined with SRIM [7], the best choices for the values of all other parameters are not completely satisfactory:

- Experimentally fitted theoretical values for $C_{e}$ available in [24] span a very narrow range of temperatures (800 K to $1600 \mathrm{~K}$ for Si, $500 \mathrm{~K}$ to $1200 \mathrm{~K}$ for Ge whereas temperature ranges attainable in our $\mathrm{MD}$ simulations go from $300 \mathrm{~K}$ to $80000 \mathrm{~K}$ ).

- Two values different by nearly a factor three for $\gamma_{p}$ have been used for Monte-Carlo simulations of SHI irradiation in $\mathrm{Si}[25,26]$.

- The best methods to determine the $t_{e p h}$ and $v_{0}$ parameters are still subject to debate $[11,17]$.

In this work, a parametric statistical study of the $\gamma_{s}$, $\gamma_{p}, C_{e}, v_{0}$ and $t_{e p h}$ TTM parameters impact on collision cascades both in $\mathrm{Si}$ and Ge materials is performed by running TTM-MD simulations initiated by $10 \mathrm{keV}$ PKAs. This study seeks to:

( $i$ ) Assess the sensitivity to the TTM parameters of the number of defects at the end of the cascade and of the PKA penetration depth in Si and in Ge materials.

(ii) Describe how the TTM parameters act on the physics of collision cascades in two materials very distinct by their thermal properties.

All the different TTM sets of parameters tested are called "scenarios" and compared to a "reference scenario" whose parameter values rely on the experimental and theoretical values available and on our previous studies [5, 27].

The methodology for the simulation of TTM-MD collision cascades as well as the TTM model, TTM parameters of interest and their value in the reference scenario are presented in section 2. Sensitivity of the results to the TTM parameters is studied for various scenarios both in Si and Ge materials in section 3. Discussions on the physics behind the observed impacts of the parameters are presented in section 4 .

\section{Methods}

\subsection{Setting up the $M D$ simulations}

To initiate the collision cascade, the PKA is given a velocity corresponding to $10 \mathrm{keV}$ and a certain direction. It shocks other atoms on its path, creating Secondary Knockon Atoms (SKAs), which displace other atoms from their equilibrium positions as well. The stochasticity of the so created cascades makes it mandatory to run a large number of simulations, each initiated by a PKA with a distinct initial direction. 100 simulations per set of parameters have been necessary to work with meaningful statistical data.

The TTM-MD simulations have been run using the LAMMPS code [28]. Boxes of 1000000 atoms made of $50 \times 50 \times 50$ cubic diamond unit cells are employed in most directions, but for some channelling PKAs directions, boxes of 4096000 atoms made of $80 \times 80 \times 80 \mathrm{cu}-$ bic diamond unit cells are necessary. The simulation box is divided into two areas: the outer cells (1 unit cell of width, i.e $5.431 \AA$ ) form an NVT ensemble in which the atomic displacements are controlled by a Langevin thermostat (damping time of $0.1 \mathrm{ps}$ ) to maintain the temperature at a desired value, and the inner cells form an NVE ensemble in which the atoms evolve freely to simulate the collision cascade.

The initialization of the simulation is made by scaling the velocities of all atoms, such that the overall temperature is the desired one, $100 \mathrm{~K}$ in the current work. The system is equilibrated during 20 ps with a timestep of $1 \mathrm{fs}$.

The maximum velocity of the atoms drastically changes between each step of the cascade: fast during the collision part, and slower during the thermal wave and recrystallization part [5]. Consequently, it is possible to decrease 
the precision of the integration timestep in parallel of the velocities, while maintaining a displacement lower than $0.025 \AA$ between two steps. In the present work, running $10 \mathrm{keV}$ simulations with $\mathrm{Si}$ and Ge on $1 \mathrm{~ns}$, the typical timesteps are: 0.01 fs during $2 \mathrm{ps}$, then 0.1 fs from 2 ps to 20 ps, and finally 1 fs between 20 ps and 1 ns.

Stillinger-Weber types potentials are employed for the simulations both in $\mathrm{Si}$ and Ge. For Si, the SW potential employed is the one developed in [29], and for Ge the one parametrized in [30] with the slight modifications made in [2]. However, to better describe short interatomic distances and thus collisions, the SW potential must be combined to a stronger repulsive two-body potential. The repulsive potential used is the Ziegler Biersack Littmark one (ZBL) [31] for both Si and Ge. SW and ZBL potentials are combined together through a Fermi function as in [32]:

$$
V_{t o t}(r)=(1-F(r)) V_{Z B L}(r)+F(r) V_{S W}(r)
$$

where $V_{t o t}$ is the total potential, $V_{Z B L}$ the repulsive $\mathrm{ZBL}$ potential, $V_{S W}$ the SW potential and $F(r)$ the Fermi function used to link the two potentials. The expression of the Fermi function of Eq. 1 as well as its parameters values for $\mathrm{Si}$ and Ge can be found in [27].

The thermal properties of the SW potentials used for $\mathrm{Si}$ and Ge are not modified by the changes in the repulsive region. Thermal properties both for the Si and the Ge potential, which are of prime importance in this study, are presented and compared to experimental values in Table 1 . The values are the ones reported by Lopez et al. in [4]. The melting temperatures show great consistency with experimental values. Simulated thermal conductivities are overestimated both for $\mathrm{Si}$ and $\mathrm{Ge}$, but the ratio, important for discussion, between $\kappa_{S i}$ and $\kappa_{G e}$ in the experimental and simulated case is conserved. Thus, despite the discrepancies between the experimental and simulated values, the qualitative observations are not jeopardized.

Table 1: Experimental (Expt.) and simulated (Simu.) values of the melting temperatures $T_{m}$ and thermal conductivities $\kappa($ at $500 \mathrm{~K}$ ) with the employed potentials for Si [29] and Ge [2, 30]. The values displayed are the ones reported in [4].

\begin{tabular}{lcccc}
\hline \hline & \multicolumn{2}{c}{ Si } & \multicolumn{2}{c}{ Ge } \\
\hline & Expt. & Simu. & Expt. & Simu. \\
\hline$T_{m}(\mathrm{~K})$ & 1685 & 1653 & 1211 & 1211 \\
$\kappa(\mathrm{W} / \mathrm{cm} / \mathrm{K})$ & 0.78 & 1.74 & 0.33 & 0.72 \\
\hline \hline
\end{tabular}

\subsection{The Two-Temperature model and key parameters}

Based on the theoretical studies conducted on the role of electronic effects in collision cascades by Caro et al. [6] and Koponen [33], Duffy et al. proposed a way of combining the TTM to MD simulations of radiation damage [8].

It consists in coupling the ionic system to an electronic subsystem made of an homogeneous electron gas as illustrated in Fig. 1. Both the ionic system and the electronic subsystem are divided in finite element cells, that form two

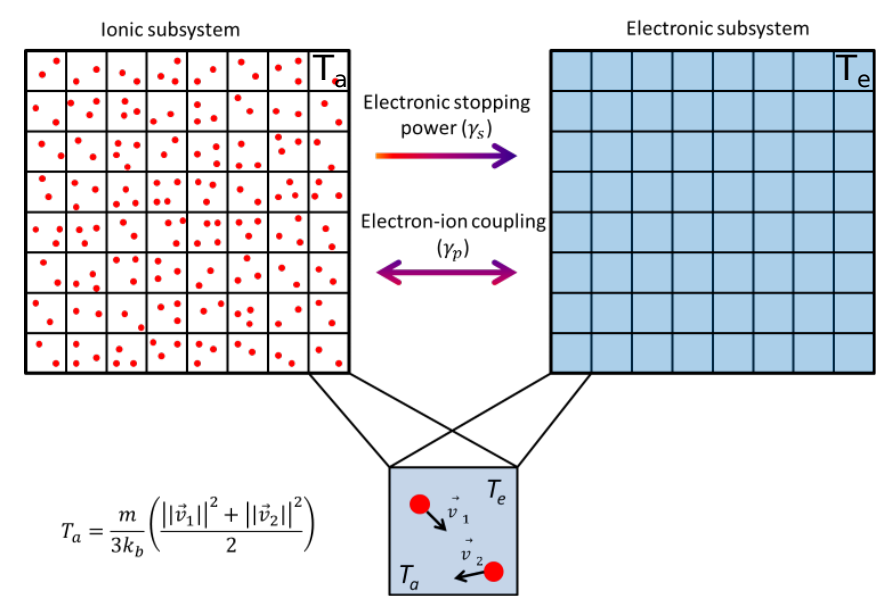

Figure 1: 2D scheme of the working principle of the TwoTemperature Model. Red dots represent atoms.

grids with the same size. In each cell, an atomic temperature $T_{a}$ and an electronic temperature $T_{e}$ are defined. In practice, each cell must contain enough atoms to define a statistically meaningful temperature. In our simulations, a grid of 20x20x20 corresponding to an average of 125 atoms per cell ensures that the electronic and atomic temperatures are not subject to harsh oscillations.

The finite elements cells of the ionic and electronic systems which are at the same position exchange energy through $\gamma_{s}$ and $\gamma_{p}$ as pictured in Fig. 1. The ions lose a part of their kinetic energy because of $\gamma_{s}$ and $\gamma_{p}$. This energy is transferred to the electronic subsystem, which increases its temperature $T_{e}$. Part of the energy gained by the electronic subsystem can be fed back to the ionic system through the electron-ion coupling $\gamma_{p}$. The ionic equations of motion used in MD are thus completed by a friction term which removes energy, and by a stochastic force term which gives back the energy as in Eq. 2:

$$
m_{i} \vec{a}_{i}=\vec{F}_{i}^{a d i a b}-\gamma_{i} \vec{v}_{i}+\vec{F}_{i}^{s t o c h}
$$

where, $m_{i}$ is the mass of the ion $i, \vec{v}_{i}$ is the velocity of the ion $i, \vec{a}_{i}$ is the acceleration of the ion $i, \vec{F}_{i}^{a d i a b}$ represents the adiabatic forces acting on the atom $i$ deriving from the potential, $\gamma_{i}$ is the friction coefficient, and $\vec{F}_{i}^{\text {stoch }}$ represents the stochastic forces acting on the atom $i$ and depends on the electronic temperature.

The $\gamma_{i}$ coefficient depends on the velocity of the considered ion. If the velocity of the ion $i$ is below a critical velocity $v_{0}$, then $\gamma_{i}=\gamma_{p}$, otherwise, $\gamma_{i}=\gamma_{s}+\gamma_{p}$. The friction coefficient $\gamma_{i}$ can then be defined like in Eq. 3:

$$
\begin{cases}\gamma_{i}=\gamma_{p} & \text { if }\left\|\vec{v}_{i}\right\| \leq v_{0} \\ \gamma_{i}=\gamma_{p}+\gamma_{s} & \text { if }\left\|\vec{v}_{i}\right\|>v_{0}\end{cases}
$$

Moreover, it is often recommended to let the system evolve for a fraction of ps before including the electronion coupling [11] because during the early stages of the 
cascade, the atomic temperatures are ill-defined due to the very fast-moving atoms. We added this option in LAMMPS through the definition of a new parameter called $t_{e p h}$.

In addition, the heat diffusion in the electronic subsystem is taken into account via the following relation:

$$
C_{e} \frac{\partial T_{e}}{\partial t}=\nabla\left(\kappa_{e} \nabla T_{e}\right)-g_{p}\left(T_{e}-T_{a}\right)+g_{s} T_{a}^{\prime}
$$

where, $C_{e}$ is the electronic specific heat, $T_{e}$ is the electronic temperature, $\kappa_{e}$ is the electronic heat conductivity, $T_{a}$ is the atomic temperature, $T_{a}^{\prime}$ has the dimension of a temperature and contributes to the energy balance, $g_{s}$ and $g_{p}$ are coefficients deriving from $\gamma_{s}$ and $\gamma_{p}$ respectively.

The TTM parameters we investigate in this paper are $C_{e} \gamma_{s}, \gamma_{p}, v_{0}$ and $t_{e p h}$. All those parameters as well as their values or expressions for the reference scenario of the parametric study of section 3 are given respectively in paragraphs $2.2 .1,2.2 .2,2.2 .3,2.2 .4$ and 2.2.5.

\subsubsection{Electronic specific heat $\left(C_{e}\right)$}

The electronic specific heat $C_{e}$ describes the amount of energy needed to increase by $1 \mathrm{~K}$ the temperature of the electronic system. It is electronic temperature dependent. In metals, $C_{e}$ is linear at low $T_{e}$ and constant and equal to the free electron gas value of $3 / 2 k_{B}$ at high $T_{e}$. Simple models can be built to accurately describe the $T_{e}$ dependence of $C_{e}$ in the TTM for metals [8]. For semiconductors, $C_{e}$ is first nearly constant and equal to zero at low $T_{e}$ due to the band gap, and then start increasing. Khara et al. have calculated $C_{e}$ for $\mathrm{Si}$ [13]: they have estimated the $T_{e}$ dependence of $C_{e}$ in Si with Density Functional Theory (DFT) calculations. They use a Fermi-Dirac smearing for performing finite electronic temperature calculations. The purpose of this study being to assess the influence of the $C_{e}$ parameter, an approximate expression is sufficient. Thus, $C_{e}$ is considered constant and equal to the free electron gas value of $3 / 2 k_{B}$ above the Fermi temperature (when the conduction band is populated).

Aiming at constructing a simple model, we have modified the $C_{e}$ model of Duffy et al. [8] to adapt it to semiconductors in a similar manner as what was done by Phillips et al. in [15]:

$$
C_{e}(x)=0.5 \times(A-\epsilon) \times\left(1+\tanh \left(B\left(x-x_{f}\right)\right)\right)+\epsilon
$$

where $x=T_{e} / 1000, A=3 / 2 k_{B}$, and the coefficients B and $x_{f}$ are fitted to reproduce the experimental measurements [24] at low temperature and the $3 / 2 k_{B}$ asymptote above the Fermi temperatures. The Fermi temperatures are $6498 \mathrm{~K}$ and $3829 \mathrm{~K}$ for Si and Ge respectively. Due to numerical limitations, $C_{e}$ cannot be set as close to zero as desired. This is the purpose of $\epsilon$ in Eq. 5 that guarantees a minimum small value for $C_{e}\left(\epsilon=10^{-6} \mathrm{eV} / \mathrm{K}\right)$. Therefore, $C_{e}$ does not match perfectly the low temperature experimental points as can be seen in Fig. 2 and is set as low as possible, without any consequences on the results.

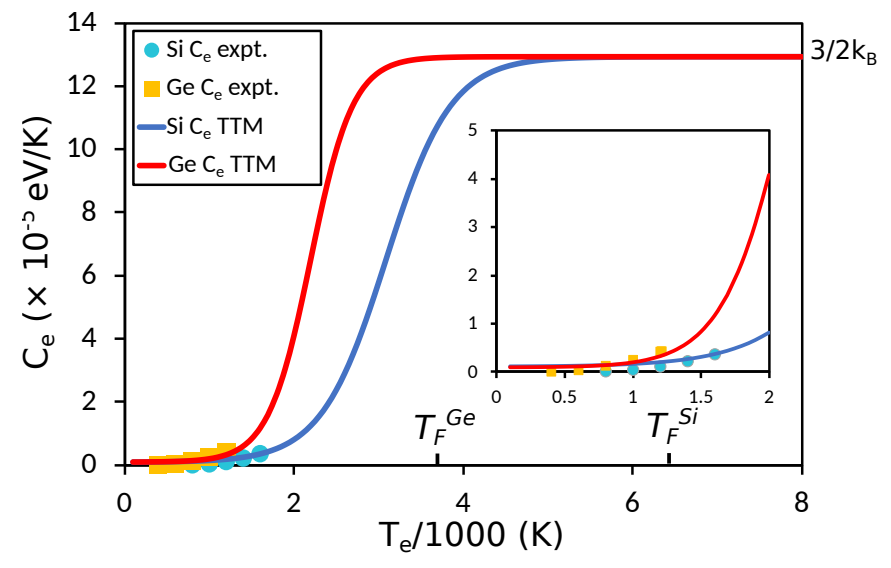

Figure 2: Variation of the electronic specific heat for $\mathrm{Si}$ and $\mathrm{Ge}$ as a function of the temperature. The Fermi temperatures, written $T_{F}^{S i}$ and $T_{F}^{G e}$, and the asymptotic value are shown. Variations at low temperatures are given in the insert.

\subsubsection{Electronic stopping power $\left(\gamma_{s}\right)$}

The electronic stopping power is a friction coefficient through which fast enough moving atoms lose energy to the electronic subsystem. It describes the excitation of the electrons due to an inelastic collision with an energetic atom. In practice, calculating the electronic stopping power of an ion moving with a certain velocity can be done with Time Dependent Density Functional Theory techniques (TD-DFT) [34]. However, the easiest way to quantify it for the TTM remains the use of SRIM tables and the Lindhard and Sharff model, as done in this study [7, 35]. For nearly $0 \mathrm{keV}$ to $10 \mathrm{keV}$ Si ions in Si, the calculation of $\gamma_{s}$ with SRIM gives $39.43 \mathrm{~g} / \mathrm{mol} / \mathrm{ps}$. For nearly $0 \mathrm{keV}$ to $10 \mathrm{keV}$ Ge ions in Ge it gives 31.64 $\mathrm{g} / \mathrm{mol} / \mathrm{ps}$. The electronic stopping power has a mechanistic effect on the moving ions by making them lose energy. Therefore the moving ions deposit less energy through nuclear elastic collisions and penetrate less deep in the material. The outcomes of changes in this parameter are of prime interest (see section 3).

\subsubsection{Electron-ion coupling $\left(\gamma_{p}\right)$}

This parameter has two roles. First it is part of the friction coefficient through which the ions lose energy to the electronic subsystem. Second, it drives the amount of energy fed back to the ions from the electronic subsystem, the energy at stake being proportional to the electron-ion coupling.

Two assumptions are made in this study. First, the electron-ion coupling parameter is considered to be equal to the electron-phonon one obtained at low temperatures in ordered systems [8]. Second, it is considered to be constant for the entire range of temperature.

For some material, the electron-phonon coupling is determined by combining experiments and theoretical models. As noticed by Duffy [8], the range of reported values for the electron-phonon coupling parameter for a given ma- 
terial can be very large. For Si, two values, differing by a factor of 3 , have been reported [25, 26]. For our simulations, unless said otherwise below, we have chosen the value of $24.443 \mathrm{~g} / \mathrm{mol} / \mathrm{ps}$ [25] which was optimized for SHI simulations in $\mathrm{Si}$ to provide the best fit with experimental data. No data were available for the electron-phonon coupling in Ge, we have therefore chosen to use the same as in Si. The values for the two materials are expected to be similar considering the common properties they share. The current hypothesis is that the real Ge value is probably slightly lower than the Si one due to the enhanced size of thermal spikes in Ge compared to Si ones which could make it harder for Ge to support phonons [8].

\subsubsection{Velocity threshold for the electronic stopping power $\left(v_{0}\right)$}

The electronic stopping power is in the theory described as acting on ions whose velocity is not too small [36]. At low energies, only the electron-phonon coupling acts on the ions [37]. The $v_{0}$ parameter idea is to incorporate this fact into the TTM model. The choice of a correct value or rule to follow for $v_{0}$ is still debated: Zarkadoula et al. [18] and Jay et al. [5] used a threshold corresponding to twice the cohesive energy of the material whereas Björkas et al. [38] employed a threshold velocity corresponding to the cohesive energy and Nordlund et al. [39] chose the value of $10 \mathrm{eV}$. In order to be coherent with our previous studies $[5,27]$, we choose to set $v_{0}$ to twice the cohesive energy of the system. Thus, $v_{0}$ is set to $79.76 \AA / \mathrm{ps}$ (i.e. a cohesive energy of $4.63 \mathrm{eV}[40]$ ) in $\mathrm{Si}$ and $45.20 \AA / \mathrm{ps}$ (i.e. a cohesive energy of $3.85 \mathrm{eV}$ [40]) in Ge.

\subsubsection{Electron-phonon activation time $\left(t_{\text {eph }}\right)$}

It is recommended to turn on the electron-phonon coupling only after a certain amount of time [11]. Indeed, in the early stages of a cascade, the atomic temperatures $T_{a}$ calculated by the MD code are artificially high due to the presence of very fast moving atoms. Thus, the energy exchanges between the electronic and ionic subsystems are biased at the beginning of the cascade. This issue can be overcome by delaying the electron-phonon energy exchanges. We added this option in LAMMPS, thus defining a new parameter called $t_{e p h}$. The electron-phonon coupling $\gamma_{p}$ is then:

$$
\begin{cases}\gamma_{p}=0 & t \leq t_{e p h} \\ \gamma_{p}=24.443 \mathrm{~g} / \mathrm{mol} / \mathrm{ps} & t>t_{e p h}\end{cases}
$$

\section{Results}

To investigate the effects of the TTM parameters, 100 simulations are performed for each TTM parameterization. For a better understanding, each case is given a "scenario" name:
- The Reference (Ref) scenario refers to a case where the default $\gamma$ settings defined in section 2 are employed $\left(\gamma_{p}=24.443 \mathrm{~g} / \mathrm{mol} / \mathrm{ps}, \gamma_{s}=39.43 \mathrm{~g} / \mathrm{mol} / \mathrm{ps}\right.$ for Si and $\gamma_{s}=31.64 \mathrm{~g} / \mathrm{mol} / \mathrm{ps}$ for $\mathrm{Ge}$ ) with a $C_{e}$ varying as shown in Fig. 2. The threshold $v_{0}$ is set to $79.76 \AA / \mathrm{ps}$ for $\mathrm{Si}$ and $45.20 \AA / \mathrm{ps}$ for Ge (i.e. the velocity corresponding to twice the cohesive energy of each material). This scenario is considered to be the baseline scenario to which all the others are compared.

- The electronic friction (ElFr) scenario corresponds to a Ref scenario where $\gamma_{s}$ is set to $100 \mathrm{~g} / \mathrm{mol} / \mathrm{ps}$.

- The electron-phonon (EL-PH) scenario corresponds to a Ref scenario where $\gamma_{p}$ is set to $100 \mathrm{~g} / \mathrm{mol} / \mathrm{ps}$.

- The High $\mathbf{C}_{\mathbf{e}}$ scenario corresponds to a Ref scenario where $\mathrm{C}_{\mathrm{e}}$ is set constant and equal to the high $\mathrm{T}$ asymptotic value of $3 / 2 \mathrm{k}_{\mathrm{B}}$.

- The Low $\mathbf{C}_{\mathbf{e}}$ scenario corresponds to a Ref scenario where $\mathrm{C}_{\mathrm{e}}$ is set constant and equal to a low value of $10^{-6} \mathrm{eV} / \mathrm{K}$.

- The Low $\mathbf{v}_{\mathbf{0}}$ scenario corresponds to a Reference scenario where $v_{0}$ is set to the velocity corresponding to once the cohesive energy, i.e. $56.39 \AA / \mathrm{ps}$ for Si and $32.12 \AA / p s$ for Ge.

- The phonon (PH) keyword might be written next to the Ref (i.e. Ref (PH)), Low $C_{e}$ and High $C_{e}$ scenario names. It means the $t_{e p h}$ parameter is switched on to the value of $0.25 \mathrm{ps}$.

To rightfully compare the electron-phonon and electronic friction scenarios, the sum $\gamma_{i}$ of $\gamma_{s}$ and $\gamma_{p}$ is kept constant between the two scenarios. Thus, when $\gamma_{p}$ is equal to $100 \mathrm{~g} / \mathrm{mol} / \mathrm{ps}, \gamma_{s}$ is set to the default value of $\gamma_{p}$ equal to $24.443 \mathrm{~g} / \mathrm{mol} / \mathrm{ps}$. Therefore, $\gamma_{i}=\gamma_{s}+\gamma_{p}$ is always equal to $124.443 \mathrm{~g} / \mathrm{mol} / \mathrm{ps}$ for the ElFr and EL-PH scenarios, for both Si and Ge materials. For the Ref, Ref (PH), ElFr and EL-PH scenarios, the properties investigated in the cascade are the PKA penetration depth, the number of defects at the end of the cascade and the maximum electronic temperature. For the High $C_{e}$, High $C_{e}(\mathrm{PH})$, Low $C_{e}$, Low $C_{e}(\mathrm{PH})$ and Low $v_{0}$ scenarios, the number of defects at the end of the cascades and the maximum electronic temperature are investigated. Indeed, it was not judged relevant to compare the PKA depth observed in those scenarios because $C_{e}$ and $v_{0}$ have no friction effect on the moving ions. A large variety of methods to count defects have been compared by Nordlund et al. in [2]. It was found that all the methods scale really well one with each other. In this study, the Lindemann criterion technique is used [41]. This method and parameters values for $\mathrm{Si}$ and Ge are described in [27]. At each timestep of a MD simulation the number of interstitials and vacancies are equal. Thus, the number of defects is taken as the number 
of vacancies (or interstitials), not the sum of the two. The mean values of all those parameters for each simulation scenario are displayed in Table 2.

\subsection{Production of damage and PKA penetration depth in the ElFr and EL-PH scenarios}

In this section, the results of the ElFr and EL-PH scenarios are compared to the Ref one in order to evaluate the impact of $\gamma_{s}$ and $\gamma_{p}$.

The mean numbers of defects observed at the end of the simulations are given in Table 2, and their distributions in Fig. 3(a) and Fig. 3(b). According to Table 2, this number in $\mathrm{Si}$ (resp. in Ge) decreases from 622 (resp. 2361) for the Ref case, to 416 (resp. 1569) in the electronic friction scenario and 410 (resp. 1338) in the electron-phonon scenario. In the same way, Fig. 3(a) and Fig. 3(b) exhibit a drastic decrease of the number of defects when each of the two $\gamma$ parameters increase. This implies that the $\gamma$ parameters play a fundamental role in the reduction of the number of final defects. However, while $\gamma_{s}$ and $\gamma_{p}$ seem to have the same influence on the reduction of the number of defects in $\mathrm{Si}(416 \approx 410)$, this reduction is enhanced in the electron-phonon scenario in Ge $(1569>1338)$.

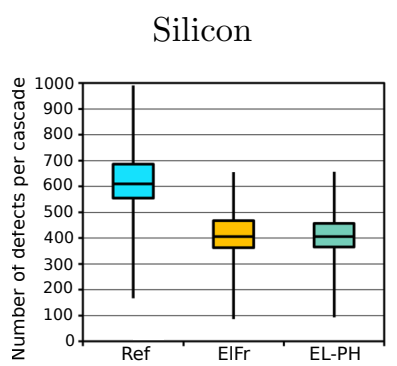

(a) Number of defects

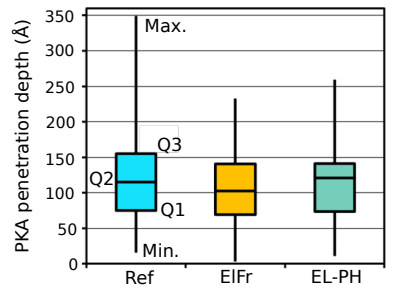

(c) PKA depth
Germanium

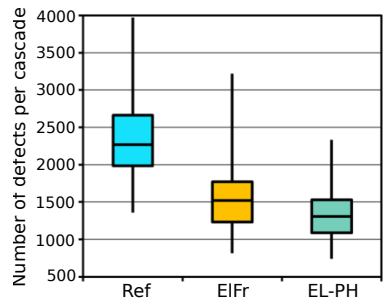

(b) Number of defects

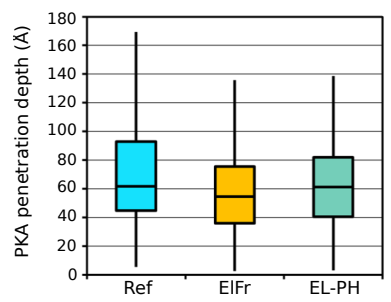

(d) PKA depth
Figure 3: Box plots of the number of defects at the end of the simulations for the Ref and $\gamma$ scenarios in Si (a) and Ge (b). Box plots of the PKA penetration depths for the Ref and $\gamma$ scenarios in Si (c) and Ge (d). The lowest horizontal black bars correspond to the first quartile values (Q1), the middle ones to the median values (Q2) and the third ones to the third quartiles values (Q3). The two black vertical bars below and above each box represent the minimum (Min.) and maximum (Max.) values of the datasets. These box plots are constructed from 100 simulations per scenario.

The mean penetration depth of the PKAs is given in Table 2, and their distributions in Fig. 3(c) and Fig. 3(d). There are no clear downward shift neither in the PKA depths distributions of Fig. 3(c) and Fig. 3(d) nor in the mean PKA depth values of Table 2. The maximum difference between all the mean PKA depths of Table 2 is less than $15 \AA$ both for $\mathrm{Si}$ and Ge. It is thus difficult to identify a global behavior in this case.

Hence, both $\gamma$ parameters do not seem to play a major role in the size of the cascade at the energy of interest $(10 \mathrm{keV})$. The only doubtlessly observable change is the reduction in the maximum PKA depth when moving to the electronic stopping and electron-phonon scenarios. The reason is that the maximum PKA penetration depths are observed when the PKA is in a channeling direction where very small amount of energy is lost through shocks. In these specific directions, the PKA is then more subject to the influence of the TTM. Even if no trends can be identified with $10 \mathrm{keV}$ ions, the $\gamma$ parameters are expected to have an important role in the penetration depth at higher energies.

\subsection{Damage production in the Ref, Low $C_{e}$ and High $C_{e}$ scenarios}

The mean number of defects that were obtained with the Ref, Low and High $C_{e}$ scenarios are given in Table 2 and the associated distributions are displayed in Fig. 4(a) for Si and Fig. 4(b) for Ge. There is a clear downward shift of the entire defect distributions in Fig. 4(a) and Fig. 4(b) from the Low $C_{e}$ to Ref scenarios for $\mathrm{Si}$ and $\mathrm{Ge}$. In Ge, the number of defects decreases again from the Ref to the High $C_{e}$ scenario. On the contrary in $\mathrm{Si}$, no difference is visible between the Ref and High $C_{e}$ scenarios. Following Table 2, in $\mathrm{Si}$ (resp. Ge), the mean number of defects decreases from 699 (resp. 2625) in the Low $C_{e}$ scenario to 618 (resp. 2191 ) in the High $C_{e}$ scenario. The mean number of defects calculated in the Ref scenario lies exactly between the two extreme scenarios for Ge whereas it lies at the same level as the High $C_{e}$ scenario in Si. The effects of $C_{e}$ are therefore more important in Ge than in Si.

\subsection{Influence of $v_{0}$ on the number of defects}

The impact of a change in $v_{0}$ between the two most recommended rules (i.e. one time the cohesive energy [5, 18] for the Low $v_{0}$ scenario or two times the cohesive energy [38] for the Ref scenario) to set it is investigated. The mean number of defects that were obtained with the Reference and Low $v_{0}$ scenarios are displayed in Table 2. In $\mathrm{Si}$, there is only a discrepancy of 2 defects between the Ref (622) and Low $v_{0}(624)$ scenarios whereas the SEM is of 13 in each scenario. In Ge, the discrepancy is of 11 defects (2361 for the Ref scenario and 2372 for the Low $v_{0}$ scenario) but the SEM is of 58 in each case. Thus, there is not any difference between the Ref and the Low $v_{0}$ scenarios at the considered energies of $10 \mathrm{keV}$.

\subsection{Influence of $t_{\text {eph }}$ on the number of defects}

The effect that $t_{e p h}$ has on the previously observed trends is investigated here. Table 2 gives the mean values of the number of defects for the $C_{e}$ scenarios with and 
Table 2: Mean values of the PKA depth, of the number of defects at the end of the cascade and of the maximum electronic temperature. Average obtained over 100 simulations for all the scenarios considered. In parenthesis are given the standard errors of the mean. $E_{c o h}$ corresponds to the cohesive energy and $m$ to the mass.

\begin{tabular}{|c|c|c|c|c|c|c|c|c|c|c|}
\hline & \multirow[b]{2}{*}{ El-ph coupling } & \multicolumn{2}{|c|}{$C_{e}$ as in Fig. 2} & \multirow{3}{*}{$\begin{array}{c}\text { High } \gamma_{s} \\
\text { Always } \\
\text { ElFr }\end{array}$} & \multirow{3}{*}{$\begin{array}{l}\text { High } \gamma_{p} \\
\text { Always } \\
\text { EL-PH }\end{array}$} & \multicolumn{2}{|c|}{$C_{e} \approx 0$} & \multicolumn{2}{|c|}{$C_{e}=3 / 2 \mathrm{k}_{B}$} & \multirow{3}{*}{$\begin{aligned} & v_{0}= \sqrt{2 \times E_{\text {coh }} / m} \\
& \text { Always } \\
& \text { Low } v_{0}\end{aligned}$} \\
\hline & & Always & After $0.25 \mathrm{ps}$ & & & Always & After $0.25 \mathrm{ps}$ & Always & After $0.25 \mathrm{ps}$ & \\
\hline & Scenario name & Ref & $\operatorname{Ref}(\mathrm{PH})$ & & & Low $C_{e}$ & Low $C_{e}(\mathrm{PH})$ & High $C_{e}$ & $\operatorname{High} C_{e}(\mathrm{PH})$ & \\
\hline \multirow{3}{*}{$\mathrm{Si}$} & PKA depth $(\AA)$ & $120(7)$ & & $107(5)$ & $114(5)$ & & & & & \\
\hline & Defects & $622(13)$ & $620(11)$ & $416(9)$ & $410(8)$ & $699(14)$ & $676(14)$ & $618(12)$ & $614(9)$ & $624(13)$ \\
\hline & $\operatorname{Max} T_{e}(\mathrm{~K})$ & 7439 & 7525 & 11619 & 11152 & 121548 & 150594 & 4905 & 4812 & 7477 \\
\hline \multirow{3}{*}{$\mathrm{Ge}$} & PKA depth $(\AA)$ & $70(5)$ & & $59(3)$ & $62(3)$ & & & & & \\
\hline & Defects & $2361(58)$ & $2396(61)$ & $1569(44)$ & $1338(33)$ & $2625(63)$ & $2643(61)$ & $2191(53)$ & $2075(49)$ & $2372(58)$ \\
\hline & $\operatorname{Max} T_{e}(\mathrm{~K})$ & 3875 & 3691 & 5584 & 5932 & 78121 & 83638 & 1768 & 1777 & 3690 \\
\hline
\end{tabular}

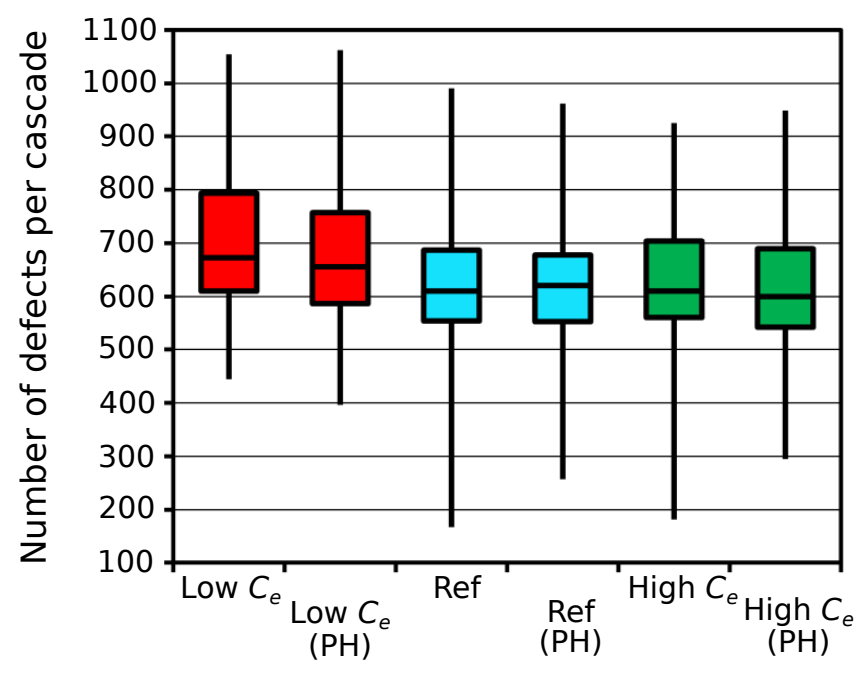

(a) Silicon

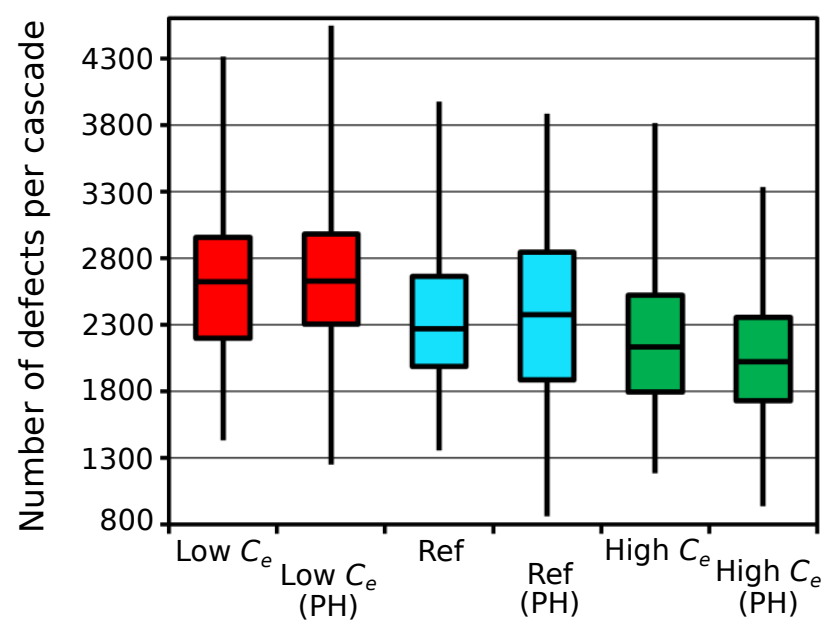

(b) Germanium

Figure 4: Box plots of the number of defects in $\mathrm{Si}$ and Ge at the end of the MD simulations for different $\mathrm{C}_{\mathrm{e}}$ scenarios. Results are provided based on 100 simulations per scenario.

without $t_{e p h}$ and Fig. 4(a) and Fig. 4(b) the global distributions. According to Table 2 the differences in the mean values of defects between the $C_{e}$ scenarios with or without $t_{e p h}$ are at most 25 defects in $\mathrm{Si}$. In Ge, the differences are less than 50 defects for the Ref and Low $C_{e}$ scenarios whereas it is a bit higher, 120 defects, for the High $C_{e}$ scenario. Considering the global distributions of Fig. 4(a) in $\mathrm{Si}$ for the same $C_{e}$ scenarios with or without $t_{e p h}$, the differences are too small to identify a trend. In Ge, no difference is visible with or without $t_{\text {eph }}$ in the global distributions of Fig. 4(b) except for the High $C_{e}$ (phonon) scenario which seems to have undergone a slight decrease compared to the High $C_{e}$ (without $t_{e p h}$ ) scenario. On the whole, the hierarchy between the $C_{e}$ scenarios in terms of number of defects is unchanged and the differences observed with the use of $t_{e p h}$ are so small that $t_{e p h}$ does not seem to play any role.

\section{Discussions}

Three main experimental trends are confirmed by the TTM-MD simulations:

- Experimental studies show that a lot more defects are observed in Ge than in Si [20, 21]. Those trends are confirmed by our own simulations in which in the Ref scenario there are in average about $300 \%$ more defects in Ge (Fig. 3(b)) than in Si (Fig. 3(a)).

- Experiments have shown that the penetration depth of various ions is higher in Si than in Ge [42]. Our simulations confirm that self PKAs penetrate always deeper in Si (Fig. 3(c)) than in Ge (Fig. 3(d)).

- It was also shown experimentally that amorphous regions are preferentially formed in Si-Ge alloys and in pure Ge than in pure Si [21]. Our simulations tend to confirm this phenomenon. In Table 3 is displayed the distribution of the defects inside the clusters depending on the cluster sizes for both $\mathrm{Si}$ and Ge in the Ref scenarios. It is found that there are less clusters of defects in Ge (mean value of 21 in Ge compared to 34 in $\mathrm{Si}$ ) and that the vast majority of defects in Ge are contained within very large amorphous pockets of defects. On the contrary, in $\mathrm{Si}$, the clusters formed are of much smaller size which indicates they are not part of large amorphous pockets. 
Table 3: Average number of clusters created by a $10 \mathrm{keV}$ PKA at the end of 100 TTM-MD simulations in Si and Ge materials for Ref scenario, and the distribution of the defects inside these clusters as a function of their size.

\begin{tabular}{lcc} 
a function of their size. & $\mathrm{Si}$ & $\mathrm{Ge}$ \\
\hline \hline Mean number of clusters & 34 & 21 \\
$\%$ of defects in clusters of size $\leq 5$ & $11 \%$ & $3 \%$ \\
$\%$ of defects in clusters of size $>5$ and $\leq 100$ & $57 \%$ & $5 \%$ \\
$\%$ of defects in clusters of size $>100$ and $\leq 1000$ & $32 \%$ & $15 \%$ \\
$\%$ of defects in clusters of size $>1000$ & $0 \%$ & $77 \%$ \\
\hline \hline
\end{tabular}

Heat spikes, or thermal spikes image the local melting of a material under the effect of elastic and inelastic interactions between an incoming energetic particle and the atoms of the target material. The hot liquid zones which are formed during a thermal spike may form amorphous regions when they cool down. MD simulations have helped understanding that the heat spike mechanism is the leading mechanism in the formation of large amorphous pockets in Ge. On the contrary, in Si, even if some heat spikes can lead to amorphous pockets, a much larger portion of small clusters remains [2, 4, 43]. The lower melting point of Ge compared to $\mathrm{Si}$ as well as its lower thermal conductivity, which makes the dissipation of heat slower, were cited as the two main reasons for the enhanced melting of Ge in response to particle irradiation [4]. The melting point and the thermal conductivity are both determined by the interatomic potential. Therefore, the fine tuning of the thermal properties of the interatomic potential is of prime importance.

\subsection{Si versus Ge: different mechanisms in response to particle irradiation}

To finely understand the effects of the TTM in Si and Ge, we plotted in Fig. 5 the average evolution of the number of TTM ionic cells that have an atomic temperature higher than the melting temperature of the material (melting temperature of $1687 \mathrm{~K}$ for $\mathrm{Si}$ and $1211 \mathrm{~K}$ for Ge) over 100 simulated cascades. Very quickly, the curves reach their peak value (collision phase), then, rapidly decrease for a very short time before continuing to slowly decrease. The fact that a cell has a temperature higher than the melting one does not mean it is melted. To be melted, cells must necessary have a temperature higher than the melting point, for a sufficient amount of time. In the following, we refer to hot cells as the ones having a temperature higher than the melting temperature. The number of hot cells combined with the time for the relaxation of the hot cells are relevant indicators of the presence of molten regions in the material.

We observe a clear difference in Fig. 5(a) and Fig. 5(b) between $\mathrm{Si}$ and Ge: the number of hot cells is always higher in $\mathrm{Ge}$ and the relaxation time required for the number of hot cells to reach zero is longer.

However, as explained in the use of $t_{e p h}$ (see paragraph 2.2 ), during the early stages of a cascade the calculated
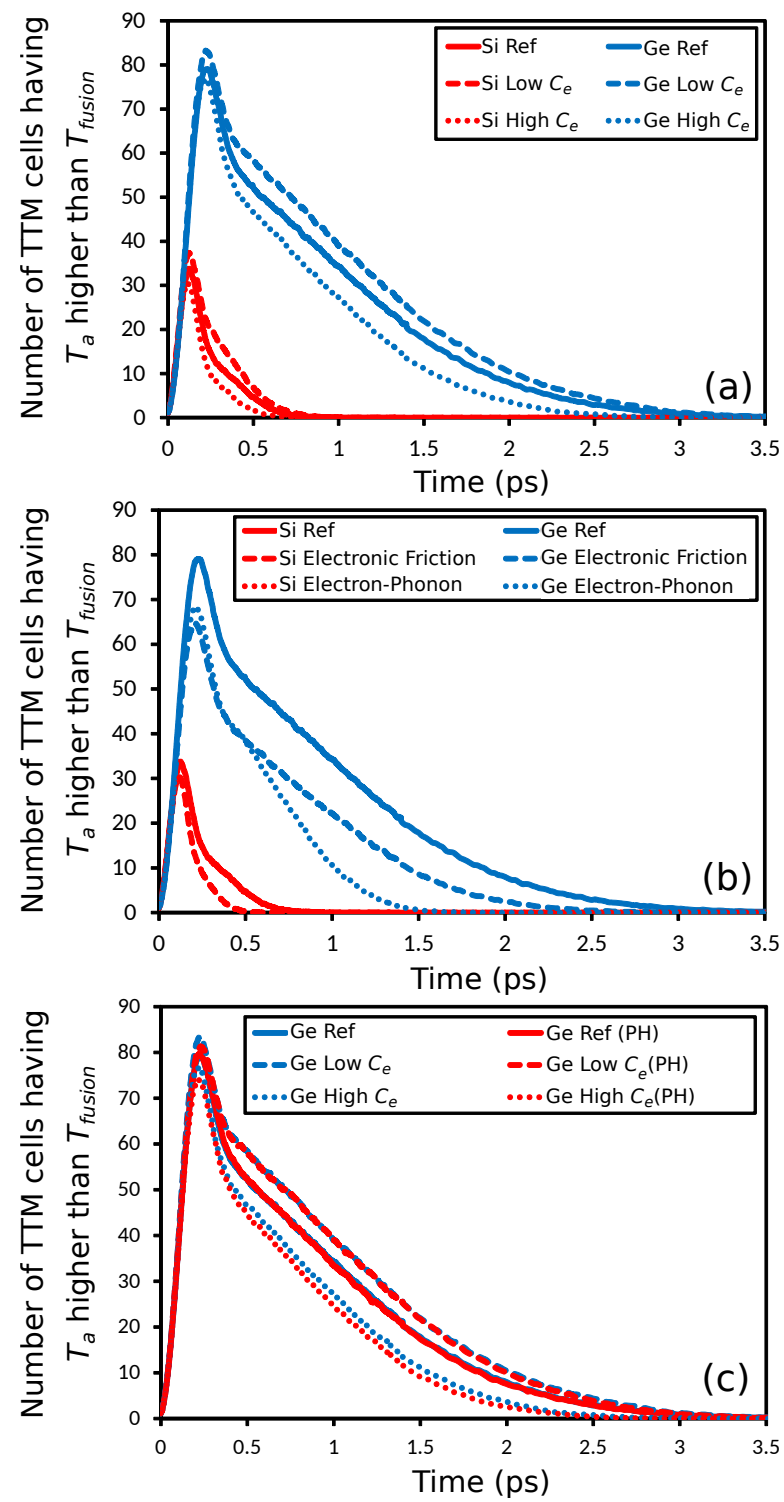

Figure 5: Evolution of the number of TTM cells having a temperature higher than the melting temperature (a) in Si and Ge for the three different $C_{e}$ scenarios (b) in $\mathrm{Si}$ and Ge for all the $\gamma$ parameters scenarios (c) in Ge for the $C_{e}$ scenarios with and without the $t_{e p h}$ option. Each curve is the result of an average over 100 simulations.

temperature does not represent an accurate evaluation of the temperature of the cell. In fact, the temperature is an average over a high number of atomic velocities, whereas the early stages of the cascade only involve the displacement of a small number of SKA. Thus, one may ask if the number of melted cells is not biased during the collision part. Generally, after $t_{e p h} \sim 0.25 \mathrm{ps}$, the collision part of the cascade is over and the fast-moving atoms have lost all their energy through shocks. This implies that about all the atoms in a same cell have the same averaged velocity and that a temperature can be well defined in this cell. Thus, only after $t_{e p h}$, Fig. 5 really shows the number of hot cells inside the materials.

The larger peak values and relaxation times for Ge sce- 
narios in Fig. 5(a) and Fig. 5(b) explain why steady amorphous pockets (hot cells becoming molten cells) are preferentially formed in Ge and not in Si. According to the mean PKA penetration depth of Si and Ge (Table 2), Ge PKAs deposit their energy in smaller regions and therefore create denser cascades and melted zones. This together with the lower thermal conductivity of Ge compared to Si (Table1) could explain the long relaxation time needed for the hot cells to lower their energy. Hence, it confirms that melting of the material is indeed far more important in Ge than in Si.

However, it is impossible to state yet that melting can partially be the result of the TTM and the electron-phonon coupling.

\section{2. $C_{e}$ scenarios reveal electron-phonon coupling partici- pates to melting within the TTM}

The hot cells are defined after the collision phase when the velocities are below $v_{0}$. In this case, the energy exchanges $\Delta U$ between the ionic subsystem and the electronic subsystem at each timestep is [8]:

$$
\Delta U=\frac{\Delta t N 3 k_{b} \gamma_{p}}{m}\left(T_{e}-T_{a}\right)
$$

where $N$ is the number of atoms in the cell, $k_{b}$ is the Boltzmann constant, $\Delta t$ is the MD timestep, $m$ is the mass of an atom, $T_{e}$ is the electronic temperature and $T_{a}$ the atomic temperature.

Thus, the more $T_{e}$ is higher than $T_{a}$ the more the energy transfer is in favor of the ionic subsystem. Moreover, in Table 2, the maximum electronic temperatures increase with $C_{e}$ decreasing. Hence, according to Eq. 7 the amount of energy gained by the ionic subsystem increases with $C_{e}$ decreasing. It is also observed in Fig. 5(a) that for both $\mathrm{Si}$ and Ge, the number of hot cells is higher for the scenarios with a lower $C_{e}$. However, $C_{e}$ does not have any effects in the rate of energy loss of the moving ions: the only mean by which a change in $C_{e}$ can induce a change in the number of hot cells is from the energy transfer from the electrons to the ions through electron-phonon coupling. The evolution of the number of hot cells in Fig. 5(a) is linked to the evolution of the mean number of defects in Table 2. It confirms our hypothesis that, in the TTM, the electron-phonon coupling is sufficient to induce partial melting of the material. The effects of $C_{e}$ are more visible in Ge than they are in $\mathrm{Si}$ because the formation of thermal spikes preferentially occurs due to interatomic potential related properties: a lower melting temperature and a lower thermal conductivity compared to Si.

One may ask if noticeable discrepancies would be observed if a DFT calculated $C_{e}$ was used (like in the work of Khara et al. [13]) instead of the $C_{e}$ of the Ref scenario. The differences between the $C_{e}$ parameters in the Low $C_{e}$, High $C_{e}$ and Ref scenarios being much more significant than what would be the differences between a DFT calculated $C_{e}$ and the $C_{e}$ of the Ref scenario, the hypothetical observed discrepancies would be small. Also, we have seen in section 3 that the number of defects is more sensitive to changes in $C_{e}$ in Ge than it is in Si. Thus, we expect no change for Si and only a slight change for Ge.

\subsection{Competition between $\gamma_{p}$ thermal and mechanistic ef- fects}

We have discussed how the $C_{e}$ scenarios reveal that melting is possible by energy transfer through $\gamma_{p}$. However, the role of $\gamma_{p}$ is dual: on the one hand, increasing $\gamma_{p}$ increases the energy transfer from the electrons to the ions but on the other hand, increasing $\gamma_{p}$ increases the energy loss of the moving atoms by electronic friction. Thus, there is a competition between the mechanistic effects that reduce the number of defects, and the thermal effects that increase the number of defects. The drastic reduction in the number of defects observed in Table 2 when increasing $\gamma_{p}$ (EL-PH scenario) reveals that the mechanistic effects are dominating over the thermal effects.

\section{4. $\gamma_{p}$ enhanced effect in Ge due to the presence of large thermal spikes}

For $\mathrm{Si}$, the mean numbers of defects that appear in Table 2 and the global distributions shown in Fig. 3(a) reveal that the reduction of the number of defects in the EL-PH and ElFr scenarios is very similar. This is correlated with the number of hot cells presented in Fig. 5(b): in the case of $\mathrm{Si}$, the curves of the two $\gamma$ parameters scenarios overlap perfectly. This means that the two $\gamma$ parameters contribute equally to the melting of the material.

On the contrary, for Ge, the mean values of Table 2 and the global distributions of Fig. 3(d) reveal that the reduction of the number of defects is enhanced in the EL$\mathrm{PH}$ scenario compared to the ElFr one. This is correlated with the number of hot cells presented in Fig. 5(b): the electron-phonon and electronic friction curves have about the same peak value but the decrease in the electronphonon curve case is sharper and the curve quickly goes below the electronic-friction one. This means that in the TTM, $\gamma_{p}$ reduces more the melting in Ge than $\gamma_{s}$ does and consequently induces a lower number of defects.

The only difference between $\gamma_{p}$ and $\gamma_{s}$ in the way they contribute to the ionic subsystem energy loss is that $\gamma_{p}$ is always activated whereas $\gamma_{s}$ is not activated when the velocity of the atom is smaller than $v_{0}$. If this difference induces no remarkable effects in $\mathrm{Si}$, it does in Ge. In fact, in Ge, the vast majority of defects is produced in liquid-like regions cooling down to become amorphous pockets, and the atoms in these regions have a velocity lower than $v_{0}$. In this case, the $\gamma_{p}$ parameter permanently acts to reduce the energies of the atoms in those amorphous pockets and $\gamma_{s}$ does not. Therefore, the sizes of the liquid-like zones shrink quicker in the EL-PH scenario compared to the ElFr scenario. Consequently, less and smaller amorphous pockets are formed and fewer defects are obtained.

The fact that there are more liquid-like regions in Ge than in $\mathrm{Si}$ is mainly due to two of their basic properties 
defined by the interatomic potential. First, the size of the Ge atom is bigger than the Si one. Consequently, the Ge PKAs and SKAs deposit their energies quickly and in smaller regions, inducing a localised melting. Second, the melting temperature of $\mathrm{Ge}$ is much lower than the one of Si. Consequently, a PKA with the same energy leads to obtain more melted cells in Ge than in Si. The effects of the TTM is then drastically correlated to the interatomic potential.

\section{5. $v_{0}$ has no influence}

The $v_{0}$ scenarios considered show no change in the mean number of defects at the end of the cascade. At the considered energies of $10 \mathrm{keV}$, the setting of $v_{0}$ to either one time the cohesive energy or two times the cohesive energy (i.e. the rules the most employed) has no influence. However, it can be expected that it has an influence at higher PKA energies.

\section{6. $t_{\text {eph }}$ has no influence}

The use of $t_{e p h}$ induces no changes in the global trends for the $C_{e}$ and $\gamma$ scenarios, at the considered energy of $10 \mathrm{keV}$. It is confirmed by Fig. 5(c) which shows the evolution of the number of TTM cells having their temperature greater than the fusion temperature. The parameter $t_{e p h}$ is however expected to influence the results in simulations where the electronic temperatures attained are greater (due to higher PKA energies for example). We still recommend to use it even in $10 \mathrm{keV}$ simulations in order to overcome the bias induced by the badly defined ionic temperature at the beginning of the cascade.

\section{Conclusion}

An extensive statistical study aiming at assessing the impact of the main TTM parameters and truly understanding their physical role within the TTM is performed. Due to the stochasticity of the cascades, carrying out a high number of simulations is of prime importance to analyse global trends of their impact. The data presented in this article are the results of more than 2000 TTM-MD collision cascades simulations initiated with $10 \mathrm{keV}$ PKAs. The comparisons between simulations with various TTM parameter values allows us to draw five main conclusions:

(i) The electronic stopping parameter and the electronphonon coupling parameter have the larger impact on the production of defects.

(ii) The influences of the electron-phonon coupling parameter and of the electronic specific heat on the number of defects are greater for materials more subject to thermal spikes.

(iii) Changes in the threshold velocity for the electronic stopping power between the two most recommended rules to set it induces no difference in the mean number of defects. (iv) The use of a parameter to set the time of activation of the electron-phonon coupling implies no change in the trends, at the considered energies.

(v) The electron-phonon coupling contributes to increase the number of defects in materials for which the irradiation leads to molten regions.

These conclusions depend on the size of the PKA and on the melting temperature of the material under investigation, which are crucial to predict the appearance of molten regions and thus the influence of the TTM. Our work is giving very valuable quantitative data for the TTM users, as well as precious qualitative data on the role the electrons have in a collision cascade simulated with MDTTM depending on the material under investigation and therefore the cascade behaviors at stake.

\section{Acknowledgments}

The authors would like to thank Layla Martin-Samos from CNR-IOM and Fuccio Cristiano from LAAS-CNRS for fruitful discussions. This article is based upon work from COST Action TUMIEE CA17126, supported by COST (European Cooperation in Science and Technology). Calculations have been performed using HPC resources from GENCI-CCRT supercomputer at CEA, DAM, DIF, HPC resources from GENCI (Grant A0030907474) and HPC resources from CALMIP (Grant 1555). T. Jarrin, A. Jay, A. Hémeryck and N. Richard are active members of the Multiscale and Multi-Model Approach for Materials in Applied Science consortium (MAMMASMIAS consortium), and acknowledge the efforts of the consortium in fostering scientific collaboration.

\section{References}

[1] J. R. Srour and J. W. Palko, IEEE Trans. Nucl. Sc. 60, 1740 (2013).

[2] K. Nordlund, M. Ghaly, R. S. Averback, M. Caturla, T. Diaz de la Rubia, and J. Tarus, Phys. Rev. B 57, 7556 (1998).

[3] K. Nordlund, S. Zinkle, S. A.E, F. Granberg, A. R.S, R. Stoller, T. Suzudo, L. Malerba, F. Banhart, W. Weber, F. Willaime, S. Dudarev, and D. Simeone, Phys. Rev. B 512, 450 (2018).

[4] P. Lopez, L. Pelaz, I. Santos, L. Marques, and M. Aboy, J. App. Phys. 111, 033519 (2012).

[5] A. Jay, M. Raine, N. Richard, N. Mousseau, V. Goiffon, A. Hémeryck, and P. Magnan, IEEE Trans. Nucl. Sc. 64, 141 (2017).

[6] A. Caro and M. Victoria, Phys. Rev. A 40, 2287 (1989).

[7] J. Ziegler, M. Ziegler, and J. Biersack, Nulc. Instr. Meth. Phys. R. B 268, 1818 (2010).

[8] D. Duffy and A. Rutherford, J. Phys.: Cond. Matter. 19, 1 (2007).

[9] E. Zarkadoula, S. Daraszewicz, D. D.M., S. M.A., T. I.T., N. K., D. M.T., and K. Trachenko, J. Phys.: Cond. Matter. 26, 085401 (2013).

[10] E. Zarkadoula, D. D.M., , N. K., S. M.A., W. Todorov I.T. Weber, and K. Trachenko, J. Phys.: Cond. Matter. 27, 135401 (2015).

[11] E. Zarkadoula, G. Samolyuk, and W. J. Weber, J. Nucl. Mat. 490, 317 (2017). 
[12] S. Daraszewicz and D. Duffy, Nulc. Instr. Meth. Phys. R. B 303, 112 (2013).

[13] G. Khara, S. T. Murphy, S. L. Daraszewicz, and D. M. Duffy, J. Phys.: Cond. Matter. 28, 395201 (2016).

[14] C. Zhang, F. Mao, and F.-H. Zhang, J. Phys.: Cond. Matter. 25, 235402 (2013).

[15] C. Phillips, R. Magyar, and P. Crozier, J. Comput. Phys. 133, 144711 (2010).

[16] V. Pisarev and S. Starikov, J. Phys.: Cond. Matter. 26, 475401 (2014).

[17] J. Cui, Z. Zhou, B. Fu, and Q. Hou, Nulc. Instr. Meth. Phys. R. B 471, 90 (2020).

[18] E. Zarkadoula, G. Samolyuk, H. Xuen, H. Bei, and W. J. Weberab, Scr. Mat. 124, 6 (2016).

[19] R. Darkins and M. Duffy, Comp. Mat. Sci. 147, 145 (2018).

[20] T. Haynes and H. O. W., Appl. Phys. Lett. 61 (1992), 10.1063/1.107669.

[21] A. N. Larsen, J. App. Phys. 81, 2208 (1997).

[22] A. Sand, S. Dudarev, and K. Nordlund, Europhys. Lett. 103, 46003 (2013).

[23] A. Sand, K. Nordlund, and S. Dudarev, Nulc. Instr. Meth. Phys. R. B , 207 (2014)

[24] C. Glassbrenner and G. Slack, Phys. Rev. A 134, 1058 (1966).

[25] A. Chettah, H. Kucal, Z. Wang, M. Kac, A. Meftah, and M. Toulemonde, Nulc. Instr. Meth. Phys. R. B 267, 2719 (2009).

[26] O. Osmani, I. Alzaher, T. Peters, B. d'Etat, A. Cassimi, H. Lebius, I. Monnet, N. Medvedev, B. Rethfled, and M. Scleberger, Nulc. Instr. Meth. Phys. R. B 282, 43 (2012).

[27] T. Jarrin, A. Jay, M. Raine, N. Mousseau, A. Hémeryck, and N. Richard, IEEE Trans. Nucl. Sc. 67, 1273 (2020).

[28] S. Plimton, J. Comput. Phys. 117, 1 (1995).

[29] F. H. Stillinger and T. A. Weber, Phys. Rev. B 31, 5262 (1985).

[30] K. Ding and H. C. Andersen, Phys. Rev. B 34, 6987 (1986).

[31] J. Ziegler, J. Biersack, and U. Littmark, The Stopping Range of Ions in Solids, 1st ed. (Pergamon Press, N.Y, 1983).

[32] N. Chen, E. Rasch, D. Huang, E. Heller, and F. Gao, IEEE Trans. Nucl. Sc. 65, 1108 (2018).

[33] I. Koponen, Phys. Rev. B 47, 14011 (1993).

[34] A. A. Correa, Comp. Mat. Sci. 150, 291 (2018).

[35] J. Lindhard and M. Sharff, Matisk-fysiske Meddelelser det Kongelige Danske Videnskabernes Selskab 27, 15 (1953).

[36] W. Eckstein, Computer Simulation of Ion-Solid Interactions (Springer, 1991).

[37] M. Finnis, P. Agnew, and J. Foreman, Phys. Rev. B 44, 567 (1991).

[38] C. Bjorkas and K. Nordlund, Nulc. Instr. Meth. Phys. R. B 267, 1830 (2009)

[39] K. Nordlund, L. Wei, Y. Zhong, and R. S. Averback, Phys. Rev. B 57, R13965 (1998).

[40] C. Kittel, Introduction to Solid State Physics, 8th ed. (John Wiley and Sons, 2004).

[41] H. Hensel and H. M. Urbassek, Phys. Rev. B 57, 4756 (1998).

[42] Y. Kayser, P. Hönicke, D. Banas, J. Dousse, J. Hoszowska, P. Jagodzinski, A. Kubala-Kukus, S. Nowak, and M. Pajek, J. An. At. S. 30, 1086 (2015).

[43] A. Jay, A. Hémeryck, N. Richard, L. Martin-Samos, M. Raine, A. Le Roch, N. Mousseau, V. Goiffon, P. Paillet, M. Gaillardin, and P. Magnan, IEEE Trans. Nucl. Sci. 65, 724 (2018). 